\title{
AWAKE FIBREOPTIC INTUBATION FOR DRAINAGE OF A THYROID ABSCESS IN A PREGNANT LADY
}

Marianne Chee ${ }^{1}$, Qing Yuan Goh ${ }^{1}$, Theodore Wong ${ }^{1}$

${ }^{1}$ Department of Anaesthesiology, Singapore General Hospital

\section{INTRODUCTION}

We present a case of a 33-year old lady who developed a thyroid mass at 25 weeks of pregnancy. 2 FNACs were performed, which revealed abscess contents. The thyroid mass enlarged significantly post FNAC MRI Neck: $4.0 \times 3.9 \times 4.5 \mathrm{~cm}$ heterogeneous mass in the left neck with extensive oedema extending superiorly into the retropharyngeal space, and tracheal displacement to the right. She was planned for an emergency incision and drainage of her left neck abscess.

\section{METHOD}

Examination findings:

- Alert, well, not in respiratory distress, SpO2 $100 \%$ on room air

- Gravid uterus, large $5 \times 4 \mathrm{~cm}$ left neck mass

- Mallampati score 2, thyromental distance $>4$ finger breadths (FB), mouth opening $>2$ FB

- Slight decreased neck extension

She was counselled for awake fibreoptic intubation (AFOI) and general anaesthesia (GA). A pre-op Doptone performed by the obstetric team was reassuring of foetal well-being.

- Nebulized with $5 \mathrm{ml} 2 \%$ Lignocaine, given IV Glycopyrrolate $0.2 \mathrm{mg}$

- Posterior oral and nasal passages sprayed with 3 sprays of $10 \%$ Lignocaine and Cophenylcaine each

- TCl Remifentanil up-titrated to $1 \mathrm{ng} / \mathrm{ml}$

- Olympus $3.8 \mathrm{~mm}$ fibreoptic bronchoscope (FOB) introduced via the right nostril (Patient comfort, technical ease)

- Aliquots of Lignocaine $2 \%$, total $10 \mathrm{ml}$, given above, at, and below the vocal cords via the epidural catheter as the scope was advanced past the cords

- A pre-loaded size 6.5 ivory RAE tube railroaded down the FOB

Once the trachea was intubated under bronchoscopic vision and good capnography waveform was achieved, GA was induced.

Intra-operative MAP kept $>70 \mathrm{mmHg}$. Direct laryngoscopy (DL) performed at the end showed no airway swelling, bleeding or pus. Vocal cords were well visualized with the ETT in situ. Anaesthesia was reversed and she was extubated successfully.

Merocel was used to pack the right nostril as there was mild bleeding Post-operative Doptone was reassuring, and she was sent to the high dependency ward.
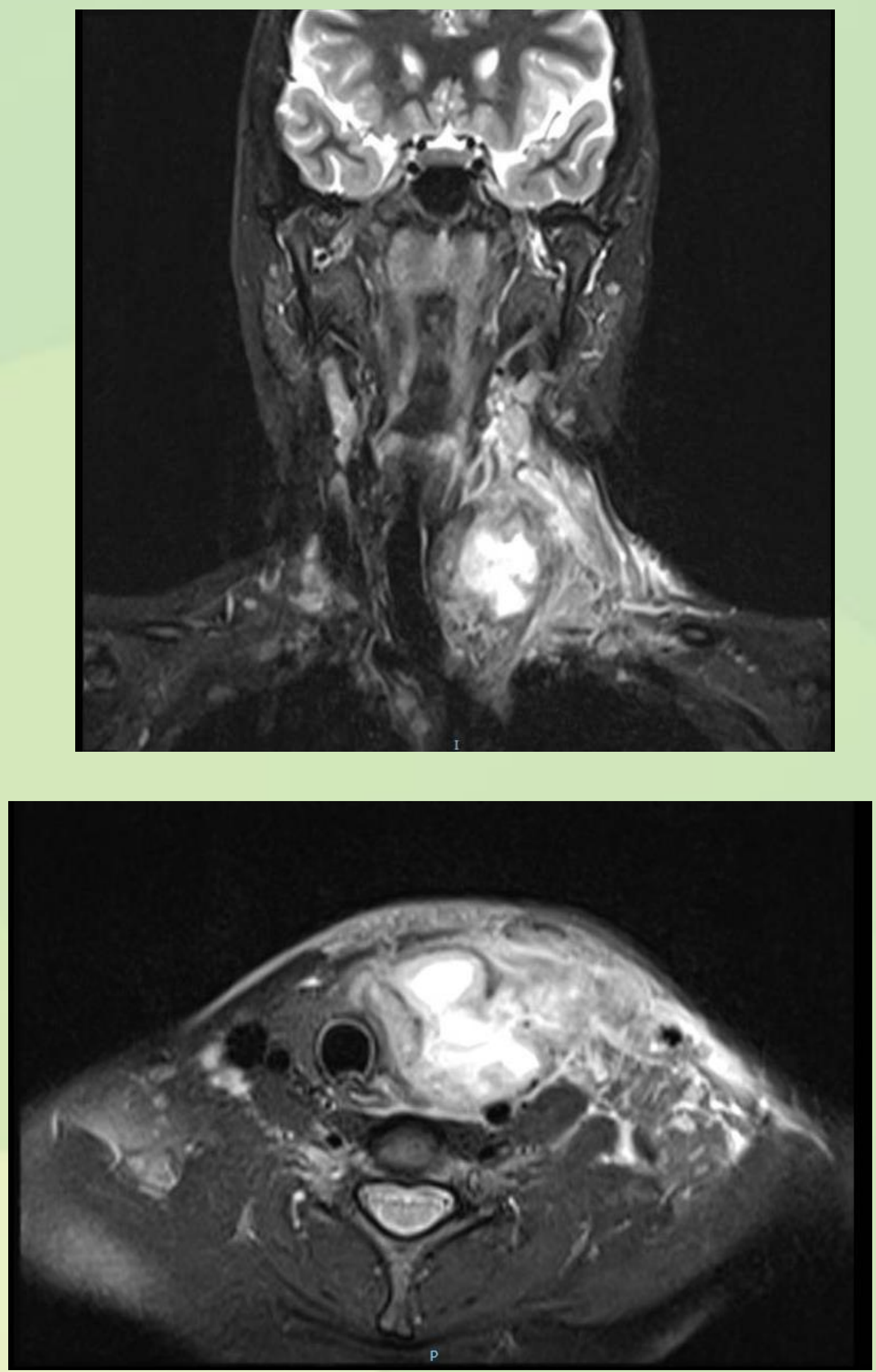

She was treated with antibiotics and wound packing was changed daily. Nasal bleeding resolved by post-operative day (POD) 2. She was discharged well on POD 14.

\section{CONCLUSION}

- AFOI should be considered in patients with possible difficult airways, especially for our patient who was both pregnant and had a large thyroid mass.

- AFOI can be performed both nasally and orally, and the nasal route is generally better tolerated and technically easier.

$>$ On hindsight, we should avoid the nasal route in peri-partum patients as the nasal passages are generally engorged.

- It is good practice to perform a DL prior to extubation to exclude airway swelling or bleeding, and to assess visibility of the vocal cords in the event patient requires intubation subsequently.

- In pregnancy, we should avoid teratogenic drugs which could possibly affect development of the fetus. We should also manage pain and anxiety to prevent pre-term labour. Consultation with an obstetric team is essential.

- Maintenance of BP peri-operatively is also paramount to ensure adequate uterine perfusion. 